\title{
OPEN Mechanically reconfigurable multi-functional meta-optics studied at microwave frequencies
}

\author{
Conner Ballew, Gregory Roberts, Sarah Camayd-Muñoz, Maximilien F. Debbas \& \\ Andrei Faraon $\bowtie$
}

\begin{abstract}
Metasurfaces advanced the field of optics by reducing the thickness of optical components and merging multiple functionalities into a single layer device. However, this generally comes with a reduction in performance, especially for multi-functional and broadband applications. Threedimensional metastructures can provide the necessary degrees of freedom for advanced applications, while maintaining minimal thickness. This work explores mechanically reconfigurable devices that perform focusing, spectral demultiplexing, and polarization sorting based on mechanical configuration. As proof of concept, a rotatable device, a device based on rotating squares, and a shearing-based device are designed with adjoint-based topology optimization, 3D-printed, and measured at microwave frequencies $(7.6-11.6 \mathrm{GHz})$ in an anechoic chamber.
\end{abstract}

Traditional optical design combines independent bulk elements to achieve complex functionality. Recent advances in nano-fabrication technologies enabled the miniaturization of bulk components by synthesizing multiple optical functionalities into single, subwavelength-thick layers called metasurfaces ${ }^{1,2}$. However, the performance of metasurfaces is limited by the number of optical modes that can be controlled, which scales with the volume of the device and the maximum refractive index contrast ${ }^{3,4}$.

Systems of metasurfaces have been used to perform functions that are beyond the capabilities of a single metasurface. A recent novel platform for this technique involves fabricating two metasurfaces on opposite sides of a transparent wafer ${ }^{5,6}$. A "folded-optics" platform uses reflective metasurfaces on both sides of a wafer to enable many interactions with different metasurfaces in a small, wafer-thick region ${ }^{7}$. This technique still uses a modular design approach, requiring the different metasurfaces be spatially separated to mitigate the unaccounted-for effects of multiple scattering events between elements that effectively invalidate the approximations commonly used in metasurface design ${ }^{8}$.

To expand the functionality of metaoptics, adjoint-based topology optimization is capable of designing highperforming dielectric structures with nonintuitive index distributions and objective functions that are challenging to achieve with traditional methods ${ }^{9,10}$. While much of the work has been applied to $2 \mathrm{D}$ platforms such as silicon photonics ${ }^{11-14}$ and flat optics ${ }^{15-17}$, free-space $2.5 \mathrm{D}$ and $3 \mathrm{D}$ devices have also been explored recently ${ }^{18-22}$. In a previous work, a passive 3D device was designed that functions as a red-green-blue (RGB) color splitter and polarizer, scaled up to microwave frequencies ${ }^{19}$. An interesting prospect for this type of device is to expand its functionality while minimizing any corresponding reduction in efficiency. For a passive device, multiple dependent functionalities will necessarily compete for efficiency. For example, the efficiency of demultiplexing spectral bins will be reduced if the device simultaneously sorts polarization. One way to maintain high efficiencies amidst multiple competing functionalities is to design active devices that can switch functionalities in time to perform the different tasks.

Previous work has explored the feasibility of coupling mechanical reconfiguration with metaoptics to enhance multi-functionality. Examples include Moiré-lens systems ${ }^{23}$, MEMS-based Alvarez lenses ${ }^{24,25}$, and computational imaging with shiftable diffractive optics ${ }^{26}$. In this work, we extend this concept by using inverse design techniques and topology optimization to design devices capable of switching between optical functions through mechanical reconfiguration. We explore 3 different methods of reconfigurability: a rotatable device that switches between three-band spectral splitting and broadband focusing when rotated $180^{\circ}$; a device based on rotating squares that performs the same functions as the rotatable device; and a device based on shearing adjacent layers that can switch between three-band spectral splitting, broadband focusing, and polarization sorting. 
(a)

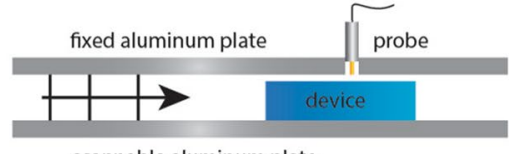

scannable aluminum plate

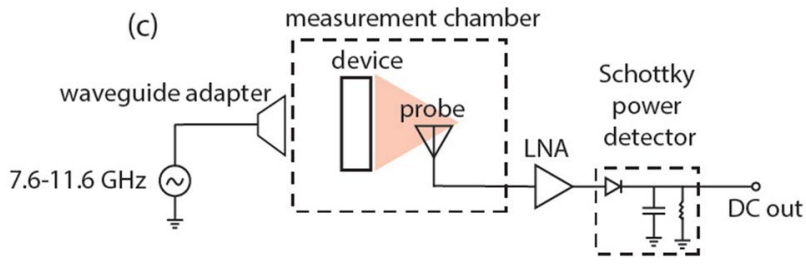

(b)

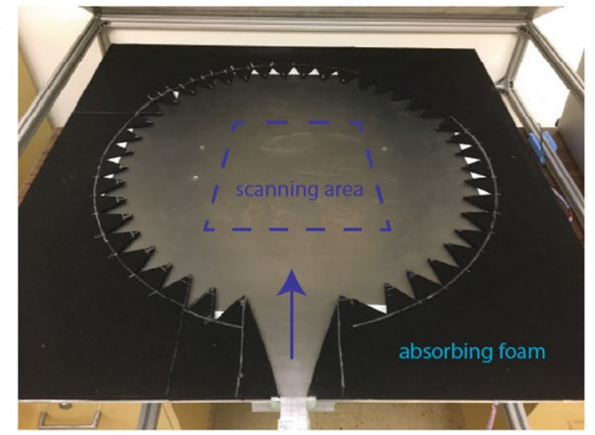

Figure 1. The anechoic chamber used to measure the 3D printed devices. (a) A sideview of the chamber. A TEM mode is injected into the anechoic chamber, exciting the device at approximately normal incidence. The fields in the cavity, including fields above the device, are measured with a small antenna probe extending through the top aluminum plate. The bottom aluminum plate is scanned in two directions to make a full $2 \mathrm{D}$ scan. (b) A picture of the anechoic chamber with the top aluminum plate removed. The scanning area is surrounded by absorbing foam. Triangles were cut out of the absorbing foam to reduce reflections. (c) A schematic representation of the measurement setup. The microwave source (Windfreak SynthHD) is scanned from 7.6 to $11.6 \mathrm{GHz}$ and is injected through a waveguide adapter. The signal from the measurement probe is first amplified with two low-noise amplifiers, and the signal is then passed through a Schottky power detector (Keysight $8470 \mathrm{~B}$ ). The output is proportional to the field amplitude in the cavity.

\section{Measurement setup and design methods}

Measurement setup. Although 3D devices can be fabricated at the micro-scale with methods such as twophoton direct laser writing and greyscale lithography, it remains challenging to fabricate fully $3 \mathrm{D}$ structures with the required features sizes and alignment accuracy for optical and near-infrared applications ${ }^{27}$. For this work, we test our ideas in the microwave range from 7.6 to $11.6 \mathrm{GHz}$ where Fused Filament Fabrication 3D-printing with polylactide acid (PLA) can generate subwavelength feature sizes with minimal material absorption. The $42 \%$ fractional bandwidth of this frequency range is the same fractional bandwidth as the range of 450-690 nm light, which is nearly the full visible spectrum.

We use a homemade anechoic microwave chamber to measure the electric fields of the devices, shown in Fig. 1. The design and construction of this setup was inspired by the work in Ref. ${ }^{28}$. The measurement system consists of a parallel plate waveguide supporting only the fundamental TEM mode in the frequency range of interest when propagating through air or PLA. A sideview schematic of the system is shown in Fig. la, and the constructed system with the top plate removed is shown in Fig. 1b. A circuit view of the system is shown in Fig. 1c. The output of a microwave synthesizer (Windfreak SynthHD) is injected into the chamber through a waveguide feed. This source is scanned from 7.6 to $11.6 \mathrm{GHz}$, which is the designed bandwidth of operation for all presented devices. A small probe antenna (SMA Connector Receptacle) is extended through the top plate of the chamber to probe the fields inside the cavity. The probe antenna signal is amplified with low noise amplifiers and propagated through a Schottky diode detector (Keysight $8470 \mathrm{~B}$ ). The resulting signal is proportional to the amplitude of the fields in the cavity. The bottom plate is moved with stepper motors to make a $2 \mathrm{D}$ scan of the field amplitude inside the chamber. The probe antenna does not extend into the cavity, so the field intensity in the small air gap above the device can be measured. These fields are approximately proportional to the fields within the device material ${ }^{28}$.

An important characteristic of this test setup is it measures $2 \mathrm{D}$ devices under TE-polarized illumination. The response of the device in the microwave chamber is nearly identical to the response of a device placed in a free space with the same index distribution, assuming the index distribution is extruded infinitely in the direction separating the waveguide plates ${ }^{28}$. This simplification from 3 to $2 \mathrm{D}$ implies we can optimize the device in a $2 \mathrm{D}$ coordinate system, thus drastically reducing the overall time of the optimization. Although these devices can be fully described by a $2 \mathrm{D}$ coordinate system, they are analogous to $3 \mathrm{D}$ free-space or $2 \mathrm{D}$ integrated photonics devices since patterning occurs in the direction of light propagation.

We design our devices assuming a refractive index of 1.5. While the actual refractive index of PLA is closer to $1.65^{29}$, the devices only fill about $80 \%$ of the gap in the microwave test chamber. This causes the effective index of the waveguide mode to shift to in between air and PLA, which we evaluate with an eigenmode simulation and plot in Supplementary Figure S1. For the designs here, we use an effective index of 1.5. There is uncertainty in this value which likely contributes to discrepancies between simulated and measured device performance. One source of the uncertainty is predicted to originate from the layered nature of the $3 \mathrm{D}$ print which has previously been reported to cause a 7\% anisotropy in PLA permittivity, with the permittivity ranging from 2.75 in the direction normal to the printing surface and 2.96 in the direction parallel to the printing surface ${ }^{29}$. Furthermore, if the permittivity depends on the layered nature of the print, then it is likely affected by the 3D printer quality and layer height, which may be different from the references used to estimate the PLA permittivity.

Topology optimization. The three designs presented are all optimized with the same strategy. The optimization technique is similar to the techniques presented in Refs. ${ }^{19,30}$, with some differences to account for the 
mechanical reconfiguration of each device, which will be briefly summarized here. The adjoint variable method is a technique to efficiently compute the gradient of a figure of merit with respect to a design region permittivity by combining the results of just two simulations, a "forward" simulation and a backward, or "adjoint", simulation. The figure of merit used here, shown in Eq. (1), is the electric field intensity at the center of the desired pixel, denoted $\left(\mathrm{x}_{0}, \mathrm{y}_{0}\right)$, so the adjoint simulation features a dipole excitation at this point ${ }^{10} . \boldsymbol{E}$ denotes the electric field vector, and $\lambda$ is the wavelength. We use a time-domain Maxwell's equations solver (Lumerical FDTD), so each simulation contains results for the entire bandwidth of interest.

$$
\operatorname{FoM}(\boldsymbol{E}, \lambda)=\left|\boldsymbol{E}\left(x_{0}, y_{0} ; \lambda\right)\right|^{2}
$$

The optimization begins with each device modelled as a block of material with a permittivity between that of air and PLA. The gradient of every figure of merit is computed at every point in the design region for each of its potential configurations. All gradients are combined with a weighted average in Eq. (3), with weights chosen according to Eq. (2) such that all figures of merit seek the same efficiency. In these equations, FoM represents the current value of a figure of merit, $N$ is the total number of figures of merit, and $w_{i}$ represents the weight applied to its respective merit function's gradient. The maximum operator is used to ensure the weights are never negative, thus ignoring the gradient of high-performing figures of merit rather than forcing the figure of merit to decrease. The $2 / N$ factor is used to ensure all weights conveniently sum to 1 unless some weights were negative before the maximum operation. Although the maximum operator is non-differentiable, this function is used only to apply the gradient rather than to compute it. Therefore, it does not affect the applicability of the adjoint method.

$$
\begin{gathered}
w_{i}=\max \left(\frac{2}{N}-\frac{F o M_{i}^{2}}{\sum F o M_{j}^{2}}, 0\right) \\
g(x, y)=\sum w_{i} \frac{\partial F o M_{i}}{\partial \epsilon}
\end{gathered}
$$

At each iteration a sub-optimization is performed to find a permittivity step that maximizes the change in the overall device performance (the product of the gradient and the permittivity step) while constrained such that the overall binarization of the device, which is a measure of how close the device is to being comprised of either air or PLA, increases with each step. This sub-optimization has the effect of increasing binarization while maximally increasing, or minimally decreasing, the device performance. We conclude the optimization by forcing each pixel to be either PLA or air which has a negligible effect on the performance of the device since the device is nearly binary by the end of the optimization. Plots showing the convergence of the figure of merit and binarization of each device are shown in Supplementary Figure S2. Once the design is finalized, the resulting permittivity distribution is exported to an .STL file and 3D printed in PLA using an Ultimaker S3.

\section{Results}

Here we describe the results from the three different devices. Measurement results feature a 2D scan of the microwave cavity chamber for each configuration, with the red-green-blue colors representing the equivalent hue of visible light when the microwave frequencies are scaled by a factor of $\times 59,618$. Although the microwave components have varying scattering parameters over the bandwidth of interest, a scan of the empty cavity is used to normalize device measurements to the total power injected into the cavity. Each device focuses to one or more of three separate pixels, depending on the desired functionality of the configuration. The pixels are depicted as either red, green, or blue, indicating the assigned color in the spectral splitting configuration with red as the lowest frequency bin $(7.6-8.9 \mathrm{GHz})$, green as the middle frequency bin $(8.9-10.2 \mathrm{GHz})$, and blue as the high frequency bin $(10.2-11.6 \mathrm{GHz})$. These frequency bins are referred to simply as red, green, or blue from now on.

The focal plane is analyzed in simulation to determine the sorting efficiency, defined as the fraction of incident power transmitting through the target pixel. To quantitatively corroborate the measured intensity profile with the simulated intensity profile, the measured devices feature a series of plots that compare the normalized intensity integrated over the frequency bands of interest for each functionality.

Rotatable device. The first device presented here changes its function by undergoing a $180^{\circ}$ rotation. The device is designed only for TE light. It focuses broadband light to the center pixel when illuminated by a normally-incident planewave from one side, and focuses red, green, and blue light to the respectively colored pixels when illuminated from the other side, as shown in Fig. $2 \mathrm{a}-\mathrm{d}$. The footprint of the device is $6.2 \mathrm{~cm} \times 18.6 \mathrm{~cm}$, which is $2 \lambda \times 6 \lambda$ at the center wavelength of $3.1 \mathrm{~cm}$, shown in Fig. 2e. To ensure structural stability the device has a thin frame of PLA, and a connectivity constraint is enforced every ten iterations of the optimization so that the index distribution converges to a fully connected structure. Connectivity is enforced by first labelling disconnected regions, then connecting them with least-cost paths (i.e. a minimum spanning tree). The cost is considered the required permittivity change to flip a voxel from low to high permittivity. In this work, we reflect the permittivity about the midpoint of air and PLA. The figure of merit does not decrease substantially due to this operation according to the convergence plots in Supplementary Figure S2.

This device is uniquely simple among the devices presented, since a $180^{\circ}$ rotation does not alter the scattering matrix beyond a transposition due to reciprocity. A reasonable concern is that this device will be limited in performance because of this. However, reciprocity does not strongly affect this device, since inputs from both sides are assumed to be normally incident. Thus, only scattering components mapping a normally incident input 

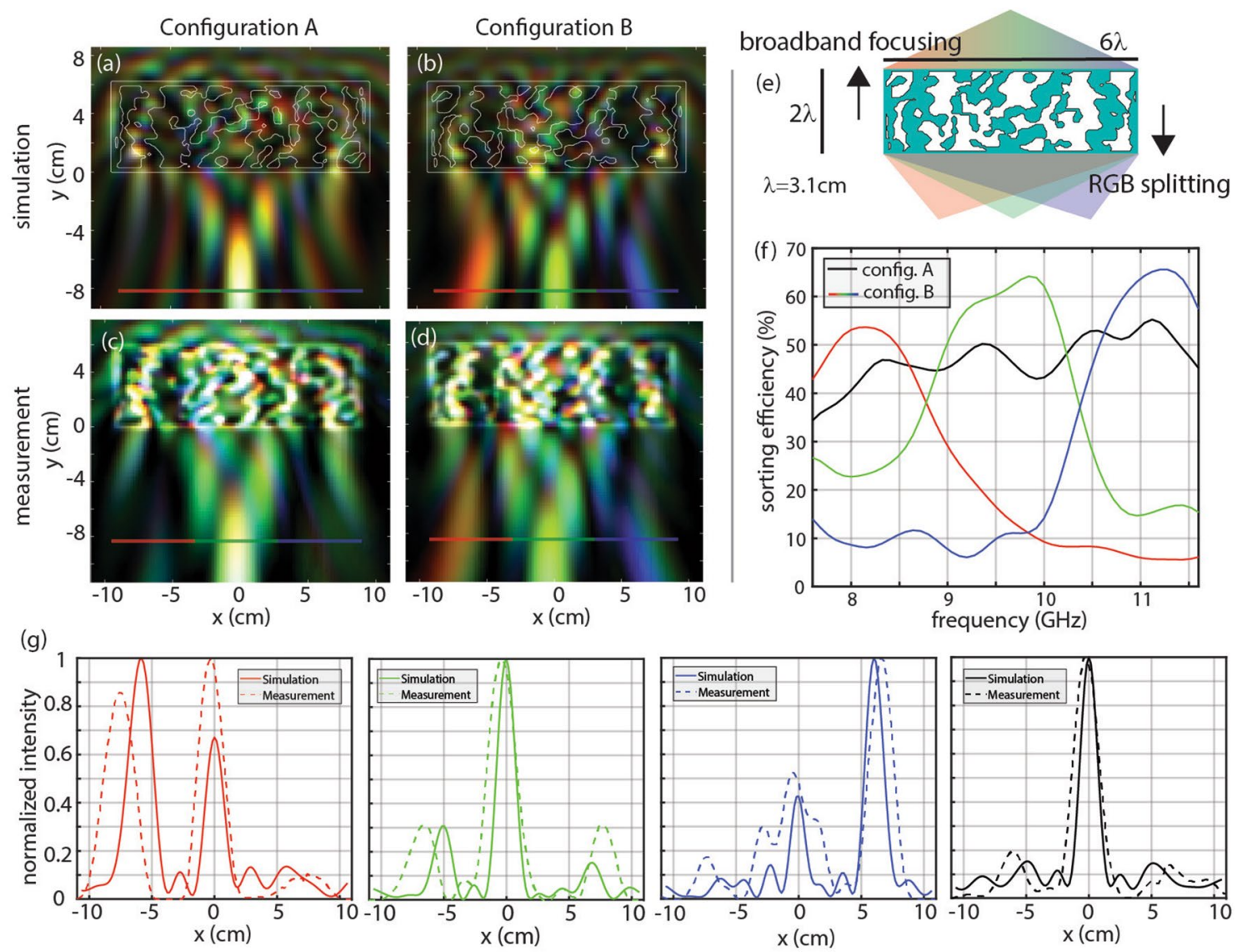

Figure 2. Rotatable device performing broadband focusing in one configuration and spectral splitting in the other configuration. (a) Broadband focusing simulation, (b) spectral splitting simulation, (c) broadband focusing measurement, and (d) spectral splitting measurement. (e) Schematic of the device, where blue represents PLA and white represents air. The footprint of the device is $6.2 \mathrm{~cm} \times 18.6 \mathrm{~cm}$. (f) Analysis of the simulated fields at the focal plane showing the sorting efficiency for each function, defined as the fraction of incident power reaching the target pixel. Red, green, and blue light are focused to their respectively colored pixels, and sorting efficiency is drawn in red, green, and blue, respectively. The broadband focusing function focuses all light to the middle pixel and is drawn in black. (g) Comparison of simulated and measurement normalized intensity profiles at the focal plane. Configuration B intensities are integrated over (left) 7.6$8.9 \mathrm{GHz}$, (center-left) $8.9-10.2 \mathrm{GHz}$, (center-right) $10.2-11.6 \mathrm{GHz}$. (right) Configuration A intensity integrated from 7.6 to $11.6 \mathrm{GHz}$.

to a normally incident output are coupled by reciprocity, while the desired output fields are comprised of many more uncoupled planewave components.

The sorting efficiency as a function of frequency is shown in Fig. 2f. The sorting efficiency averaged across the relevant frequencies for each function is $50.6 \%$. This outperforms traditional three-pixel absorptive Bayer filter arrays, which have a maximum theoretical sorting efficiency of $33 \%$ for each frequency band. To quantitatively compare the intensity profiles of measured and simulated results, normalized intensity profiles are shown in Fig. $2 \mathrm{~g}$ for each function.

Rotating squares. The second device we studied is comprised of rotatable squares. The first configuration features a $0^{\circ}$ rotation of all squares, while the second configuration features a $\pm 90^{\circ}$ rotation of each square with each square having an opposite angular rotation as its neighboring squares. This transformation can be achieved using the scheme depicted in Fig. 3e, where the squares are hinged appropriately as described in previous works ${ }^{31-33}$. A video of this transformation is available in Visualization 1.

The two functions chosen here are broadband focusing and spectral splitting for TE polarization-the same as the rotatable device. The device is fully connected, with a frame around each square element to ensure structural stability.

A total of three devices were fabricated. The first device demonstrates the transformation. To fabricate this, the $3 \mathrm{D}$-print was paused at half the thickness of the device, a nylon mesh was manually inserted, and the 3D-print was then resumed to completion. After the print, the nylon mesh was cut to leave only the required flexible hinges between each square. This device is shown in Visualization 1. The other two devices are the two different configurations printed directly, which were then measured. 

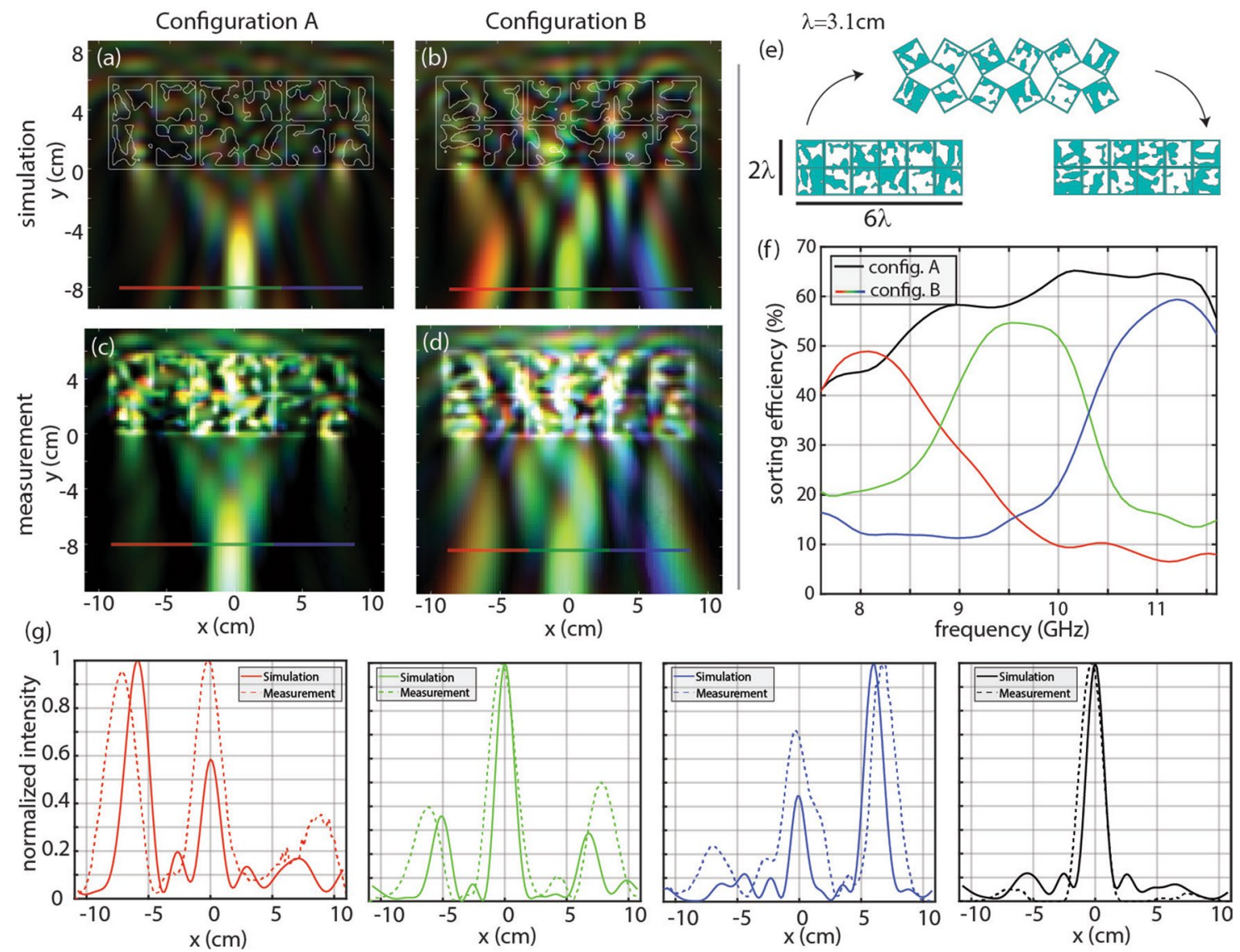

Figure 3. The device performing broadband focusing in one configuration and spectral splitting in the other configuration. (a) Broadband focusing simulation, (b) spectral splitting simulation, (c) broadband focusing measurement, and (d) spectral splitting measurement. (e) Demonstration of the transformation and device footprint, where blue represents PLA and white represents air. The footprint of the device is $6.2 \mathrm{~cm} \times 18.6 \mathrm{~cm}$. (f) Analysis of the focal plane of this device, showing the sorting efficiency for each function, defined as the fraction of incident power reaching the target pixel. Red, green, and blue light are focused to their respectively colored pixels, and sorting efficiency is drawn in red, green, and blue, respectively. The broadband focusing function focuses all light to the middle pixel and is drawn in black. (g) Comparison of simulated and measured normalized intensity profiles at the focal plane. Configuration B intensities are integrated over (left) 7.68.9 GHz, (center-left) 8.9-10.2 GHz, (center-right) 10.2-11.6 GHz. (right) Configuration A intensity integrated from 7.6 to $11.6 \mathrm{GHz}$.

This design and the rotatable design have the same device size and focal length, so the performance of the two devices can be directly compared. The simulated sorting efficiency is shown in Fig. 3f. Since the current device has a frame around each square element, less of the design area is available for optimization. This detracts from the degrees of freedom of the device, which may explain why the device has a $48.0 \%$ average sorting efficiency, $2.6 \%$ less than the rotatable device. The difference in average efficiency may also arise from the different natures of the mechanical reconfiguration. The simulated and measured intensities within the full test chamber are shown in Fig. 3a-d. To quantitatively compare the intensity profiles of measured and simulated results, normalized intensity profiles are shown in Fig. $3 \mathrm{~g}$ for each function.

Shearing device. The final device features a transformation based on shearing alternate layers. This action is achievable at the microscale through MEMS electrostatic actuation. Unlike the previous devices, this device switches between three different functionalities: spectral splitting shown in Fig. 4a, broadband focusing shown in Fig. 4b, and polarization splitting shown in Fig. 4c. All functionalities are designed for both TE and TM polarizations.

The added polarization control demands more degrees of freedom than the previous devices, and it was found that the device needed to be nearly $3 \times$ thicker than the previous devices to achieve satisfactory performance of $59.2 \%$ average sorting efficiency. This device does not have a supporting frame and does not enforce connectivity like the previous devices.

This device was analyzed only in simulation due to limitations within the measurement system: the measurement chamber only supports a TE-polarized TEM mode, and the scannable region is too small to measure this device. A 4-layer device was designed and tested with similar agreement between simulation and measurement 
(a) Config. A: RGB
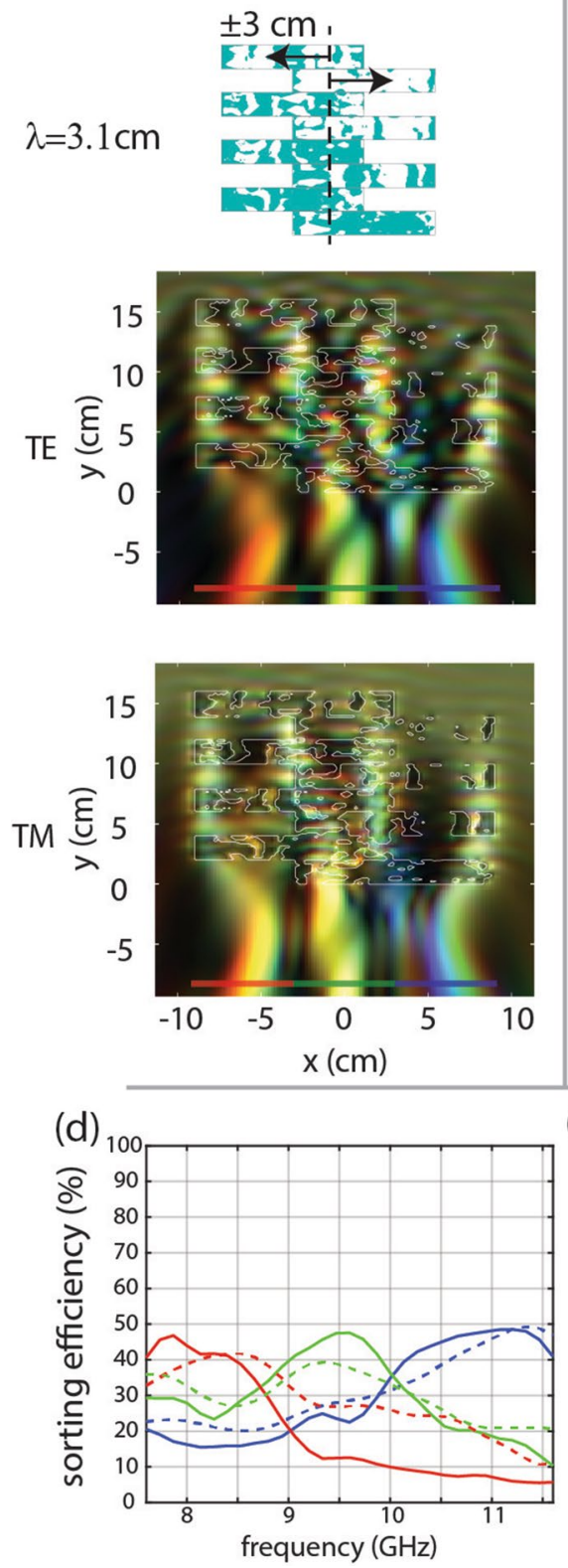

(b) Config. B: focusing
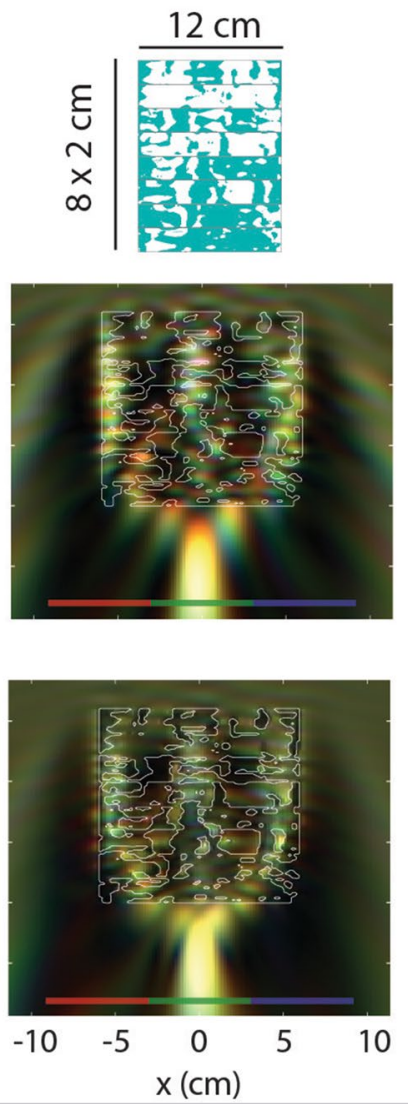

(e)

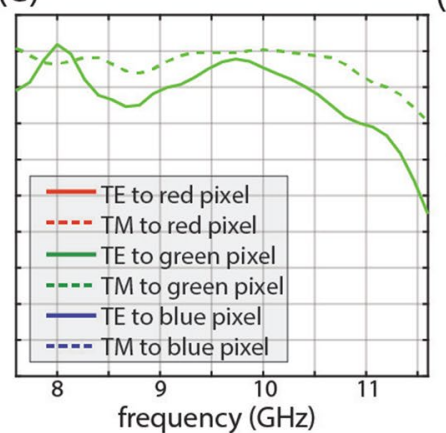

(c) Config. C: polarization
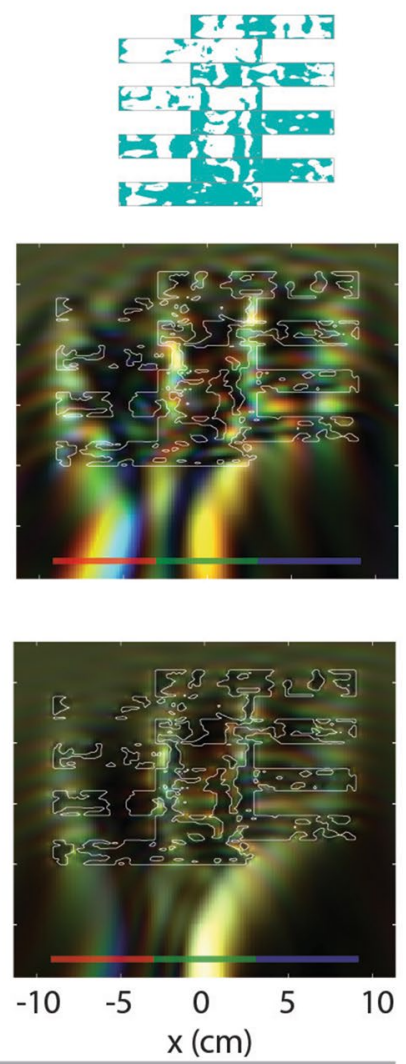

(f)

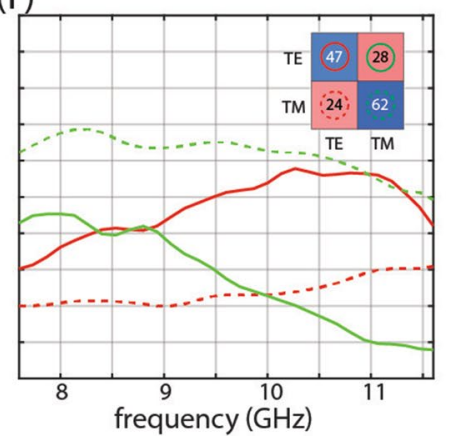

Figure 4. TE and TM fields for a device based on a net shearing movement of $6 \mathrm{~cm}$. (a) The spectral splitting configuration splits red, green, and blue light to the red, green, and blue pixels, respectively. This is analyzed for both TE and TM polarizations. (b) The neutral state of the device performs broadband focusing to the center pixel for both TE and TM polarizations. (c) The polarization sorting configuration sorts broadband TE light to the left (red) pixel and focuses broadband TM light to the center (green) pixel. (d-f) Sorting efficiency of the different configurations. The line color represents the sorting efficiency to the similarly colored pixel as depicted in the color plots. Solid lines represent the TE response, and dashed lines represent the TM response. (d) Sorting efficiency in the spectral splitting configuration. (e) Sorting efficiency for the broadband focusing configuration. (f) Sorting efficiency for the polarization splitting configuration. (Inset) A confusion matrix representation of the sorting efficiency, with true input on the vertical axis and predicted input on the horizontal axis. Each matrix entry is determined by averaging the relevant trace over the full bandwidth.

to the previous devices, but the device could not achieve all objective functions. Data for this device is available on request.

The configurations and simulation results for the 8-layer simulated device are summarized in Fig. 4 . The device is a stack of eight $8 \mathrm{~cm} \times 2 \mathrm{~cm}$ layers. The mechanical actuation displaces adjacent layers in equal and opposite directions by $3 \mathrm{~cm}$, which is approximately one wavelength. The first configuration, shown in Fig. 4a, shows spectral splitting behavior with $42 \%$ average sorting efficiency for TE and $40 \%$ average sorting efficiency for TM. The crosstalk between the different spectral bins is worse than the previous devices, with the worst case 
occurring for the TM-polarized green input which focuses only $1.2 \times$ more power to the desired green pixel than the undesired blue and red pixels.

The neutral position of the device, in which all layers are aligned as shown in Fig. 4b, features broadband focusing with high efficiency. The aperture size of this configuration is smaller than the other two configurations, and the sorting efficiency is normalized to the power incident on this smaller aperture when analyzing this configuration. The sorting efficiency shown in Fig. 4e is expected to be uniformly high across all frequencies and both polarizations.

The final configuration sorts TE polarization to the leftmost pixel and TM polarization to the center pixel. The transmission through each pixel, averaged across the entire spectrum, is summarized in matrix form in Fig. 4f: a TE input focuses $47 \%$ power to the correct pixel and $28 \%$ power to the incorrect pixel, the TM input focuses $62 \%$ power to the correct pixel and $24 \%$ power to the incorrect pixel. More power is coupled to the desired pixel than the undesired pixel at all frequencies except for the case of TE input frequencies below approximately $9 \mathrm{GHz}$. In this case more power is directed towards the incorrect green pixel than the correct red pixel. It is possible that this could be fixed by sacrificing performance in the other functionalities, such as by tuning the weighting scheme described in Eq. (1), or by increasing the thickness or index contrast of the device.

\section{Discussion}

We have studied dielectric scattering elements capable of substantially expanding their functionality through mechanical reconfiguration. The work here is a step towards answering an important question in optics-how much performance and functionality can be squeezed into a certain volume? The miniaturization of optical systems has led to new applications in lasers ${ }^{34,35}$, biomedical optics ${ }^{36,37}$, space instrumentation ${ }^{38}$, and generally applications where strict size requirements exist. Yet there is still much progress that can be made, since systems of cascaded metasurfaces still generally require some free-space propagation to preserve their independence and the validity of the techniques often employed in metasurface design. Furthermore, complex functionality such as combining polarization control, spectral splitting, and imaging into single elements has been elusive.

The approach shown here showcases the advantages of coupling mechanical reconfiguration with adjointbased electromagnetic optimization. Miniaturization is achieved by employing adjoint-based inverse design to find a locally optimal shape of a small dielectric volume, while broad multi-functional performance is achieved through mechanical reconfiguration. The designed structures are difficult to fabricate at the nanoscale for optical applications, but the design process is flexible enough to incorporate nanoscale fabrication requirements as shown in other works ${ }^{39}$. In particular, some areas of fabrication that can fabricate the required subwavelength features for these devices include: multilayer fabrication common in CMOS or MEMS processes ${ }^{40}$; direct-write two-photon laser lithography capable of designing subwavelength 3D elements in the infrared ${ }^{27}$; and closely aligned stacks of Silicon wafers with subwavelength features at terahertz frequencies ${ }^{41}$. All of these techniques have demonstrated active mechanical control that could be useful for multiplexing functions like what was demonstrated in this work.

The devices here were studied at microwave frequencies for ease of fabrication, yet the applications are particularly useful for optics. The scalability of Maxwell's equations ensures that the simulations used to compute the gradient are frequency agnostic. However, the design may need to be changed to account for different deformation and actuation mechanisms available for microscopic devices. For example, the device based on a shearing actuation presented here is analogous to electrostatically actuated devices seen in MEMS, yet considerations such as the pull-in effect would need to be carefully considered for such a device operating at the microscale ${ }^{42,43}$. Some of these challenges have been addressed in recent works that combine metaoptics with MEMS ${ }^{25,44,45}$.

Adjoint optimization is well-suited for optimizing the optical properties of mechanically reconfigurable devices. In this work, the mechanical reconfiguration scheme was predetermined, and the material was patterned to enable multi-functional performance. The performance of the rotatable device and the device based on rotating squares were comparable, suggesting that the specific choice of mechanical transformation may not drastically affect optical performance. This offers the advantage that a certain transformation can be chosen for mechanical purposes and can be subsequently patterned to imbue the device with desirable optical properties. Alternatively, the mechanical and optical properties could be co-optimized. Future work could combine existing optimization techniques for designing mechanical metamaterials ${ }^{46,47}$ with the techniques presented here, yielding devices that have co-optimized mechanical and optical performance.

Received: 19 November 2020; Accepted: 14 April 2021

Published online: 27 May 2021

\section{References}

1. Yu, N. \& Capasso, F. Flat optics with designer metasurfaces. Nat. Mater. 13, 139-150 (2014).

2. Kamali, S. M., Arbabi, E., Arbabi, A. \& Faraon, A. A review of dielectric optical metasurfaces for wavefront control. Nanophotonics 7, 1041-1068 (2018).

3. Miller, D. A. B. Fundamental limit for optical components. J. Opt. Soc. Am. B 24, A1 (2007).

4. Arbabi, A. \& Faraon, A. Fundamental limits of ultrathin metasurfaces. Sci. Rep. 7, 1-9 (2017).

5. Arbabi, A., Arbabi, E., Horie, Y., Kamali, S. M. \& Faraon, A. Planar metasurface retroreflector. Nat. Photonics 11, 415-420 (2017).

6. Groever, B., Chen, W. T. \& Capasso, F. Meta-lens doublet in the visible region. Nano Lett. 17, 4902-4907 (2017).

7. Faraji-Dana, M. et al. Hyperspectral imager with folded metasurface optics. ACS Photonics 6, 2161-2167 (2019).

8. Arbabi, A., Horie, Y., Bagheri, M. \& Faraon, A. Dielectric metasurfaces for complete control of phase and polarization with subwavelength spatial resolution and high transmission. Nat. Nanotechnol. 10, 937-943 (2015).

9. Jensen, J. S. \& Sigmund, O. Topology optimization for nano-photonics. Laser Photonics Rev. 5, 308-321 (2011). 
10. Lalau-Keraly, C. M., Bhargava, S., Miller, O. D. \& Yablonovitch, E. Adjoint shape optimization applied to electromagnetic design. Opt. Express 21, 21693 (2013).

11. Su, L. et al. Nanophotonic inverse design with SPINS: Software architecture and practical considerations. Appl. Phys. Rev. 7, 011407 (2020).

12. Hughes, T. W., Minkov, M., Williamson, I. A. D. \& Fan, S. Adjoint method and inverse design for nonlinear nanophotonic devices. ACS Photonics 5, 4781-4787 (2018).

13. Jensen, J. S. \& Sigmund, O. Systematic design of photonic crystal structures using topology optimization: Low-loss waveguide bends. Appl. Phys. Lett. 84, 2022-2024 (2004).

14. Borel, P. I. et al. Imprinted silicon-based nanophotonics. Opt. Express 15, 1261 (2007).

15. Sell, D., Yang, J., Doshay, S., Yang, R. \& Fan, J. A. Large-angle, multifunctional metagratings based on freeform multimode geometries. Nano Lett. 17, 3752-3757 (2017).

16. Pestourie, R. et al. Inverse design of large-area metasurfaces. Opt. Express 26, 33732 (2018).

17. Zhan, A. et al. Controlling three-dimensional optical fields via inverse Mie scattering. Sci. Adv. 5, eaax4769 (2019).

18. Deng, Y. \& Korvink, J. G. Topology optimization for three-dimensional electromagnetic waves using an edge element-based finiteelement method. Proc. R. Soc. A Math. Phys. Eng. Sci. 472, 20150835 (2016).

19. Camayd-Muñoz, P., Ballew, C., Roberts, G. \& Faraon, A. Multifunctional volumetric meta-optics for color and polarization image sensors. Optica 7, 280 (2020).

20. Mansouree, M. et al. Multifunctional 25D metastructures enabled by adjoint optimization. Optica 7, 77 (2020).

21. Chung, H. \& Miller, O. D. High-NA achromatic metalenses by inverse design. Opt. Express 28, 6945 (2020).

22. Callewaert, F., Velev, V., Kumar, P., Sahakian, A. V. \& Aydin, K. Inverse-designed broadband all-dielectric electromagnetic metadevices. Sci. Rep. 8, 1358 (2018).

23. Iwami, K., Ogawa, C., Nagase, T. \& Ikezawa, S. Demonstration of focal length tuning by rotational varifocal moiré metalens in an ir-A wavelength. Opt. Express 28, 35602 (2020).

24. Zhan, A., Colburn, S., Dodson, C. M. \& Majumdar, A. Metasurface freeform nanophotonics. Sci. Rep. 7, 1-9 (2017).

25. Han, Z., Colburn, S., Majumdar, A. \& Böhringer, K. F. MEMS-actuated metasurface Alvarez lens. Microsyst. Nanoeng. 6, 1-11 (2020).

26. Heide, F., Fu, Q., Peng, Y. \& Heidrich, W. Encoded diffractive optics for full-spectrum computational imaging. Sci. Rep. 6, 1-10 (2016).

27. Wei, H. et al. Two-photon direct laser writing of inverse-designed free-form near-infrared polarization beamsplitter. Adv. Opt. Mater. 7, 1900513 (2019).

28. Justice, B. J. et al. Spatial mapping of the internal and external electromagnetic fields of negative index metamaterials. Opt. Express 14, 8694 (2006).

29. Felício, J. M., Fernandes, C. A. \& Costa, J. R. Complex permittivity and anisotropy measurement of 3D-printed PLA at microwaves and millimeter-waves. in ICECom 2016-Conference Proceedings, 22nd International Conference on Applied Electromagnetics and Communications (Institute of Electrical and Electronics Engineers Inc., 2017). https://doi.org/10.1109/ICECom.2016.7843900.

30. Piggott, A. Y. et al. Inverse design and demonstration of a compact and broadband on-chip wavelength demultiplexer. Nat. Photonics 9, 374-377 (2015).

31. Grima, J. N. \& Evans, K. E. Auxetic behavior from rotating squares. J. Mater. Sci. Lett. 19, 1563-1565 (2000).

32. Grima, J. N., Zammit, V., Gatt, R., Alderson, A. \& Evans, K. E. Auxetic behaviour from rotating semi-rigid units. Phys. Status Solidi 244, 866-882 (2007).

33. Kolken, H. M. A. \& Zadpoor, A. A. Auxetic mechanical metamaterials. RSC Adv. 7, 5111-5129 (2017).

34. Arbabi, A., Briggs, R. M., Horie, Y., Bagheri, M. \& Faraon, A. Efficient dielectric metasurface collimating lenses for mid-infrared quantum cascade lasers. Opt. Express 23, 33310 (2015).

35. Xie, Y. Y. et al. Metasurface-integrated vertical cavity surface-emitting lasers for programmable directional lasing emissions. Nat. Nanotechnol. 15, 125-130 (2020).

36. Yesilkoy, F. et al. Ultrasensitive hyperspectral imaging and biodetection enabled by dielectric metasurfaces. Nat. Photonics 13, 390-396 (2019).

37. Kwon, H., Arbabi, E., Kamali, S. M., Faraji-Dana, M. S. \& Faraon, A. Single-shot quantitative phase gradient microscopy using a system of multifunctional metasurfaces. Nat. Photonics 14, 109-114 (2020).

38. Wu, Z., Li, L., Li, Y. \& Chen, X. Metasurface superstrate antenna with wideband circular polarization for satellite communication application. IEEE Antennas Wirel. Propag. Lett. 15, 374-377 (2016).

39. Piggott, A. Y., Petykiewicz, J., Su, L. \& Vučković, J. Fabrication-constrained nanophotonic inverse design. Sci. Rep. 7, 1-7 (2017).

40. Piggott, A. Y. et al. Inverse-designed photonics for semiconductor foundries. ACS Photonics 7, 569-575 (2020).

41. Jung-Kubiak, C. et al. A multistep DRIE process for complex terahertz waveguide components. IEEE Trans. Terahertz Sci. Technol. 6, 690-695 (2016).

42. Nathanson, H. C., Newell, W. E., Wickstrom, R. A. \& Davis, J. R. The resonant gate transistor. IEEE Trans. Electron Devices ED-14, 117-133 (1967).

43. Taylor, G. I. The coalescence of closely spaced drops when they are at different electric potentials. Proc. R. Soc. Lond. Ser. A Math. Phys. Sci. 306, 423-434 (1968).

44. Roy, T. et al. Dynamic metasurface lens based on MEMS technology. APL Photonics 3, 021302 (2018).

45. Arbabi, E. et al. MEMS-tunable dielectric metasurface lens. Nat. Commun. 9, 1-9 (2018).

46. Schwerdtfeger, J. et al. Design of auxetic structures via mathematical optimization. Adv. Mater. 23, 2650-2654 (2011).

47. Kaminakis, N. T. \& Stavroulakis, G. E. Topology optimization for compliant mechanisms, using evolutionary-hybrid algorithms and application to the design of auxetic materials. in Composites Part B: Engineering Vol. 43, 2655-2668 (Elsevier, 2012).

\section{Acknowledgements}

This work was funded by the Defense Advanced Research Projects Agency EXTREME program (HR00111720035).

\section{Author contributions}

C.B., S.C., and A.F. conceived the idea. C.B. and G.R. designed the devices. C.B. fabricated the devices and conducted the experiments. C.B., S.C., and M.D. constructed the measurement system. C.B. prepared the manuscript with input from all authors.

\section{Competing interests}

The authors declare no competing interests. 


\section{Additional information}

Supplementary Information The online version contains supplementary material available at https://doi.org/ 10.1038/s41598-021-88785-5.

Correspondence and requests for materials should be addressed to A.F.

Reprints and permissions information is available at www.nature.com/reprints.

Publisher's note Springer Nature remains neutral with regard to jurisdictional claims in published maps and institutional affiliations.

(c) (i) Open Access This article is licensed under a Creative Commons Attribution 4.0 International License, which permits use, sharing, adaptation, distribution and reproduction in any medium or format, as long as you give appropriate credit to the original author(s) and the source, provide a link to the Creative Commons licence, and indicate if changes were made. The images or other third party material in this article are included in the article's Creative Commons licence, unless indicated otherwise in a credit line to the material. If material is not included in the article's Creative Commons licence and your intended use is not permitted by statutory regulation or exceeds the permitted use, you will need to obtain permission directly from the copyright holder. To view a copy of this licence, visit http://creativecommons.org/licenses/by/4.0/.

(C) The Author(s) 2021 